\title{
Vector meson-baryon octet interaction and resonances generated dynamically
}

\author{
B.-X. Sun* and Y.-W. Wang \\ College of Applied Sciences, Beijing University of Technology \\ Beijing 100124, China \\ *sunbx@bjut.edu.cn
}

Published 16 April 2014

\begin{abstract}
The interaction potentials between vector mesons and baryon octet are calculated explicitly with a summation of $t-, s-$, and $u-$ channel diagrams and a contact term originating from the tensor interaction. Altogether, 13 resonances are generated dynamically in different channels of strangeness zero by solving the relativistic Lippman-Schwinger equations in the S-wave approximation, and their masses, decay widths, isospins and spins are determined. Some resonances are well fitted with their counterparts listed in the newest review of Particle Data Group (PDG), ${ }^{1}$ while others might stimulate the experimental observation in these energy regions in the future.
\end{abstract}

Keywords: Hadronic resonances; Hidden gauge symmetry; Coupled-channel unitary approach.

PACS Numbers: 14.20.Gk, 11.10.Ef, 11.15.Tk

\section{Introduction}

The combination of chiral Lagrangian with nonperturbative unitary techniques in coupled channels of mesons and baryons has become a powerful method to study the meson-meson and meson-baryon interactions and new states in the resonance region, which are not easily explained using the conventional constituent quark model. In addition, the theory on the hidden gauge symmetry supplies a mechanism to include the vector meson in the chiral Lagrangian. ${ }^{2-5}$ Therefore, the study on the interaction between vector mesons and other hadrons becomes possible. Along this clue, the interactions between the vector meson and the psuedoscalar meson are studied and the radiative decays of some axial-vector mesons generated dynamically are discussed. ${ }^{6}$ Similarly, the scattering amplitudes of two $\rho$ mesons are calculated

This is an Open Access article published by World Scientific Publishing Company. It is distributed under the terms of the Creative Commons Attribution 3.0 (CC-BY) License. Further distribution of this work is permitted, provided the original work is properly cited. 
within the framework of coupling-channel approach of the Lippman-Schwinger equation, and two resonances $f_{0}(1370)$ and $f_{2}(1270)$ are generated dynamically. ${ }^{7}$ This work has been extended to the $S U(3)$-space of vector mesons in Ref. 8, where several known resonances are also dynamically generated. In the baryon sector, the interaction of the vector meson and baryon decuplet is addressed in Refs. 9, 10, where only $t$ - channel amplitudes are analyzed in the S-wave approximation. This method is also extended to study the interaction of vector mesons and baryon octet, and several baryon resonances have been found as a result of solving the LippmanSchwinger equation in the coupled channel formalism. ${ }^{11}$ However, the resonances generated dynamically are spin degenerate since the amplitude obtained from $t-$ channel interaction is spin independent when the wave function of the initial and final baryons and the polarization vector of the incoming and outgoing mesons are all neglected. Since the masses of vector mesons are comparable to those of baryons, only $t$ - channel diagrams might be incomplete to obtain a reliable interaction of vector mesons and baryons. Thus in Ref. 12, the $t-, s-, u-$ channel diagrams and a contact diagram originating from the tensor term of the vector meson and baryon octet interaction are all taken into account, and four spin-determined resonances are found in a non-relativistic approximation. In the present work, we deduce the interaction kernel of vector mesons and baryon octet including a vector meson exchange in $t$ - channel, octet baryon exchange in $s-, u$ - channels, and a contact diagram related only to the tensor interaction term precisely, and then calculate the scattering amplitudes of vector mesons and baryon octet by solving the LippmanSchwinger equation in a coupled channel approach. In this article, we only discuss the scattering amplitude in the S-wave approximation, and we shall see that the scattering amplitudes lead to poles in the complex plane which can be associated to some well-known resonances.

In Sect. 2, we will show the basic Lagrangian obtained with the hidden gauge symmetry of $S U(3)$ group, in which a tensor interaction term is included, and then the framework on the Lippman-Schwinger equation of the coupled channel approach will be summarized briefly. The parameters in the Lagrangian are fixed according to the experimental data on coupling constants of baryon octet to vector mesons. In Sect. 3, we will show the resonances found in the complex plane for the vector mesonbaryon octet system with total strangeness zero. The masses and decay widths of these resonances and their possible PDG counterparts are also discussed. Finally, we will present a summary of this article.

\section{Formalism}

We follow the formalism of the vector meson-baryon octet interaction in Ref. 12, and then the Lagrangian for the linearly coupling to vector mesons can be explicitly written as

$$
\mathcal{L}_{V B B}=-g\left\{F_{V}\left\langle\bar{B} \gamma_{\mu}\left[V_{8}^{\mu}, B\right]\right\rangle+D_{V}\left\langle\bar{B} \gamma_{\mu}\left\{V_{8}^{\mu}, B\right\}\right\rangle\right.
$$




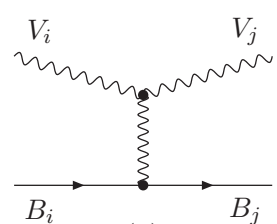

(a)

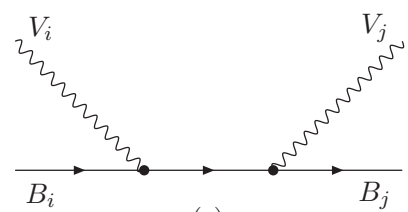

(c)

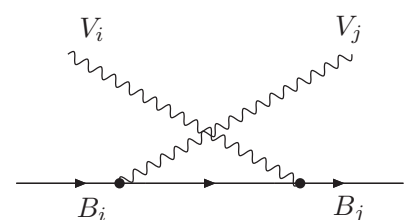

(b)

Fig. 1. Feynman Diagrams of the vector meson-baryon interaction. (a) $t-$ channel, (b) $u-$ channel, (c) $s$ - channel, and (d) contact term.

$$
\begin{aligned}
& +\frac{1}{4 M}\left(F_{T}\left\langle\bar{B} \sigma_{\mu \nu}\left[\partial^{\mu} V_{8}^{\nu}-\partial^{\nu} V_{8}^{\mu}, B\right]\right\rangle+D_{T}\left\langle\bar{B} \sigma_{\mu \nu}\left\{\partial^{\mu} V_{8}^{\nu}-\partial^{\nu} V_{8}^{\mu}, B\right\}\right\rangle\right) \\
& \left.+\frac{C_{V}}{3}\left\langle\bar{B} \gamma_{\mu} B\right\rangle\left\langle V_{0}^{\mu}\right\rangle+\frac{C_{T}}{4 M}\left\langle\bar{B} \sigma_{\mu \nu} V_{0}^{\mu \nu} B\right\rangle\right\}
\end{aligned}
$$

which will contribute to the vector meson- baryon octet interaction in $t-, s-$ and $u$ - channels. Moreover, the self-coupling terms of vector mesons lead to a contact interaction between the baryon and the vector meson, which is trivially null for singlet meson-baryon interaction, thus

$$
\mathcal{L}_{V V B B}=\frac{g}{4 M}\left\{F_{T}\left\langle\bar{B} \sigma_{\mu \nu}\left[i g\left[V_{8}^{\mu}, V_{8}^{\nu}\right], B\right]\right\rangle+D_{T}\left\langle\bar{B} \sigma_{\mu \nu}\left\{i g\left[V_{8}^{\mu}, V_{8}^{\nu}\right], B\right\}\right\rangle\right\} .
$$

From Eqs. (1) and (2), we can obtain the transition potentials for the $t-, s-$, $u$ - channels and contact interactions between baryons and vector mesons.

The scattering matrix implies solving the relativistic coupling-channel LippmanSchwinger equation in the on-shell factorization approach

$$
T(\sqrt{s}, \cos \theta)=[1-V(\sqrt{s}, \cos \theta) G]^{-1} V(\sqrt{s}, \cos \theta),
$$

with $G$ being the loop function of a vector meson and a baryon.

Because the amplitude $T$ in Eq. (3) is a function of the scattering angle $\theta$, we can expand the amplitude $T$ in a series of Legendre polynomials, i.e.,

$$
T(\sqrt{s}, \cos \theta)=\sum_{l=0}^{\infty} T_{l}(\sqrt{s}) P_{l}(\cos \theta)
$$


Table 1. The experimental values on the coupling constants of vector mesons to the baryons ${ }^{13}$.

\begin{tabular}{ccccc}
\hline Exp. & $\omega$ & $\rho$ & $K^{*}$ & $\phi$ \\
\hline$g^{2} / 4 \pi$ & 2.4 & 2.4 & 1.39 & 12.0 \\
\hline
\end{tabular}

Table 2. The parameters used in the calculation.

\begin{tabular}{cccccc}
\hline$F_{V}$ & $D_{V}$ & $F_{T}$ & $D_{T}$ & $C_{V}$ & $C_{T}$ \\
\hline 1.6405 & 0.2225 & 1.6405 & 0.2225 & -5.144 & -5.144 \\
\hline
\end{tabular}

with

$$
T_{l}(\sqrt{s})=\frac{2 l+1}{2} \int_{0}^{\pi} T(\sqrt{s}, \cos \theta) P_{l}(\cos \theta) \sin \theta d \theta,
$$

where $T_{l}(\sqrt{s})$ is the amplitude of the $l$ th partial wave. In the $S$-wave approximation, only the amplitude $T_{0}(\sqrt{s})$ is calculated for different channels.

The experimental values on coupling constants of vector mesons to baryons are listed in Table 1, which are taken from Ref. 13. Thus we can fit the parameters $F_{V}$, $D_{V}$ and $C_{V}$ in the Lagrangian with these data. Moreover, we assume the parameters $F_{T}, D_{T}$ and $C_{T}$ take the same values as $F_{V}, D_{V}$ and $C_{V}$, respectively. The values of these parameters are listed in Table 2 .

\section{Results and Discussion}

We search for poles of the scattering amplitude $\left|T_{0}\right|^{2}$ at the complex $\sqrt{s}$ plane. If the $\operatorname{Re}(\sqrt{s})$ is above the threshold of the channel, the pole might correspond a resonance state of the vector meson and the baryon. Otherwise, if $\operatorname{Re}(\sqrt{s})$ of the pole is less than the threshold, it is more possible to be a bound state. We will discuss the cases of isospin $\frac{1}{2}$ and $\frac{3}{2}$ with different spin and strangeness zero, respectively.

\section{1. $I=1 / 2, S=1 / 2$}

We find 4 poles of $\left|T_{0}(\sqrt{s})\right|^{2}$ on the complex $\sqrt{s}$ plane, which are summarized in Table 3. The pole at the position of $1620+i 77 \mathrm{MeV}$ might correspond to the resonance $N(1650) 1 / 2^{-}$in the review of the Particle Data Group. ${ }^{1}$ The poles at $2071+i 75 \mathrm{MeV}$ might have the counterpart $N(1895) 1 / 2^{-}$, which appears in the PDG review as $N^{*}(2090) S_{11}$ before $2012 .{ }^{1}$ Moreover, there are other two poles generated near $1800 \mathrm{MeV}$, and they are more close to the recent mass value of the resonance $N(1895) 1 / 2^{-},{ }^{14}$ especially, they are almost equivalent to the mass value on this resonance in earlier Refs. 15, 16. It should be pointed out that the resonances at the position of poles $1812+i 42 \mathrm{MeV}$ and $1825+i 50 \mathrm{MeV}$ couples strongly to the $\phi N$ and $K^{*} \Lambda$ channels, respectively, while that at $2071+i 75 \mathrm{MeV}$ couples to $K^{*} \Sigma$ 
Table 3. The properties of the dynamically generated resonances with isospin $I=1 / 2$ and spin $S=1 / 2$ and their possible PDG counterparts.

\begin{tabular}{llccc}
\hline Pole positions & Name and $J^{P}$ & Status & Mass & Width \\
\hline $1620+i 77 \mathrm{MeV}$ & $N(1650) 1 / 2^{-}$ & $* * * *$ & $1645-1670 \mathrm{MeV}$ & $120-180 \mathrm{MeV}$ \\
$1812+i 42 \mathrm{MeV}$ & & & & \\
$1825+i 50 \mathrm{MeV}$ & & $* *$ & $\approx 2090 \mathrm{MeV}$ & $100-400 \mathrm{MeV}$ \\
$2071+i 75 \mathrm{MeV}$ & $N(1895) 1 / 2^{-}$ & $* *$ & & \\
\hline
\end{tabular}

Table 4. Pole positions and coupling constants to various channels of the resonances found in the $I=1 / 2$ and $S=1 / 2$ sector.

\begin{tabular}{ccccc}
\hline$g_{i}$ & $1620+i 77 \mathrm{MeV}$ & $1812+i 42 \mathrm{MeV}$ & $1825+i 50 \mathrm{MeV}$ & $2071+i 75 \mathrm{MeV}$ \\
\hline$\rho N$ & $0.39-i 0.21$ & $0.012+i 0.16$ & $-0.39-i 0.26$ & $-0.027+i 0.046$ \\
$\omega N$ & $0.11+i 0.13$ & $0.42+i 0.27$ & $-0.078-i 0.17$ & $-0.02+i 0.033$ \\
$\phi N$ & $1.36+i 0.17$ & $1.41+i 0.18$ & $-0.0039-i 0.028$ & $0.001+i 0.005$ \\
$K^{*} \Lambda$ & $0.73-i 0.39$ & $1.07-i 0.31$ & $1.30+i 0.019$ & $0.0+i 0.06$ \\
$K^{*} \Sigma$ & $-0.10+i 0.029$ & $-0.18-i 0.038$ & $-0.32-i 0.0014$ & $0.52+i 0.48$ \\
\hline
\end{tabular}

mainly. The coupling constants of these resonances to baryons and vector mesons are listed in Table 4 . In a conclusion, there might be more complicated resonance distributions around the thresholds of $\phi N, K^{*} \Lambda$ and $K^{*} \Sigma$.

\section{2. $I=1 / 2, S=3 / 2$}

Altogether, we find five poles on the complex $\sqrt{s}$ plane, which are listed in Table 5 . The pole at $1651+i 9 \mathrm{MeV}$ might correspond to the resonance $N(1685) ?^{?}$, whose spin and parity are not determined. if so, the spin of these resonance might be $3 / 2$ and the parity might be negative. It is more interesting to note the poles on $1840+i 102 \mathrm{MeV}$ and $2050+i 14 \mathrm{MeV}$, which correspond to the resonances $N(1875) 3 / 2^{-}$and $N(2120) 3 / 2^{-}$, respectively. These two resonances had been labeled as one resonance $N(2080)$ before the 2012 PDG Review. There is new evidence from Ref. 14 for two $3 / 2^{-}$states exist in the region above $1800 \mathrm{MeV}$. The resonance corresponding to the pole on $1840+i 102 \mathrm{MeV}$ couples strongly to $\phi N$, while the resonance at $2050+i 14 \mathrm{MeV}$ has a larger coupling constant to $K^{*} \Sigma$, as shown in Table 6 . Moreover, we find a pole at $1722+i 5 \mathrm{MeV}$, which might correspond to the resonance $N(1700) 3 / 2^{-}$, and another pole at $1933+i 16 \mathrm{MeV}$, which has no counterpart in the PDG data. ${ }^{1}$

\section{3. $I=3 / 2, S=1 / 2$}

Three poles are found in the complex plane of $\sqrt{s}$, and these poles and their counterparts are listed in Table 7 . These three poles couple strongly to $K^{*} \Sigma$, as summarized in Table 8. It should be iterated that in Ref. 12, the authors also obtained a pole in the $2 \mathrm{GeV}$ region of the channel $I=3 / 2$ and $S=1 / 2$ using their non-relativistic 
Table 5. The properties of the dynamically generated resonances with isospin $I=1 / 2$ and spin $S=3 / 2$ and their possible PDG counterparts.

\begin{tabular}{ccccc}
\hline Pole positions & Name and $J^{P}$ & Status & Mass & Width \\
\hline $1651+i 9 \mathrm{MeV}$ & $N(1685) ?^{?}$ & $*$ & $1670-1685 \mathrm{MeV}$ & $\approx 25 \mathrm{MeV}$ \\
$1722+i 5 \mathrm{MeV}$ & $N(1700) 3 / 2^{-}$ & $* * *$ & $1650-1750 \mathrm{MeV}$ & $100-250 \mathrm{MeV}$ \\
$1840+i 102 \mathrm{MeV}$ & $N(1875) 3 / 2^{-}$ & $* * *$ & $1820-1920 \mathrm{MeV}$ & $160-320 \mathrm{MeV}$ \\
$1933+i 16 \mathrm{MeV}$ & & & & \\
$2050+i 14 \mathrm{MeV}$ & $N(2120) 3 / 2^{-}$ & $* *$ & $\approx 2120 \mathrm{MeV}$ & $\approx 300 \mathrm{MeV}$ \\
\hline
\end{tabular}

Table 6. Pole positions and coupling constants to various channels of the resonances found in the $I=1 / 2$ and $S=3 / 2$ sector.

\begin{tabular}{cccccc}
\hline$g_{i}$ & $1651+i 9 \mathrm{MeV}$ & $1722+i 5 \mathrm{MeV}$ & $1840+i 102 \mathrm{MeV}$ & $1933+i 16 \mathrm{MeV}$ & $2050+i 14 \mathrm{MeV}$ \\
\hline$\rho N$ & $0.36+i 0.63$ & $-0.022-i 0.050$ & $-0.46+i 0.48$ & $0.051+i 0.12$ & $0.013+i 0.073$ \\
$\omega N$ & $-0.14+i 0.061$ & $0.10+i 0.15$ & $0.74+i 0.023$ & $0.14-i 0.33$ & $0.019+i 0.049$ \\
$\phi N$ & $1.69-i 0.15$ & $0.67+i 0.20$ & $1.66-i 0.28$ & $1.14-i 0.38$ & $0.0048-i 0.023$ \\
$K^{*} \Lambda$ & $1.18-i 0.10$ & $-0.029+i 0.048$ & $1.12-i 0.43$ & $-0.54-i 0.48$ & $0.070+i 0.058$ \\
$K^{*} \Sigma$ & $-0.24-i 0.095$ & $-0.018-i 0.045$ & $-0.17-i 0.099$ & $0.075+i 0.12$ & $0.89+i 0.003$ \\
\hline
\end{tabular}

Table 7. The properties of the dynamically generated resonances with isospin $I=3 / 2$ and spin $S=1 / 2$ and their possible PDG counterparts.

\begin{tabular}{ccccc}
\hline Pole positions & Name and $J^{P}$ & Status & Mass & Width \\
\hline $1588+i 27 \mathrm{MeV}$ & $\Delta(1620) 1 / 2^{-}$ & $* * * *$ & $1600-1660 \mathrm{MeV}$ & $130-150 \mathrm{MeV}$ \\
$2002+i 28 \mathrm{MeV}$ & $\Delta(1900) 1 / 2^{-}$ & $* *$ & $1840-1920 \mathrm{MeV}$ & $140-300 \mathrm{MeV}$ \\
$2056+i 37 \mathrm{MeV}$ & $\Delta(2150) 1 / 2^{-}$ & $*$ & $\approx 2150 \mathrm{MeV}$ & $120-200 \mathrm{MeV}$ \\
\hline
\end{tabular}

potential between vector mesons and baryon octet, which is related to the resonance $\Delta(1900) 1 / 2^{-}$, just as we have done.

\section{4. $I=3 / 2, S=3 / 2$}

In the case of isospin $I=3 / 2$ and spin $S=3 / 2$, only one pole is found in the complex plane of $\sqrt{s}$, which might correspond to the resonance $\Delta(1700) 3 / 2^{-}$in the PDG data. Contrary to the case of spin $S=1 / 2$, this resonance couples strongly to the channel $\rho N$, as depicted in Tables 9 and 10 .

Table 8. Pole positions and coupling constants to various channels of the resonances found in the $I=3 / 2$ and $S=1 / 2$ sector.

\begin{tabular}{cccc}
\hline$g_{i}$ & $1588+i 27 \mathrm{MeV}$ & $2002+i 28 \mathrm{MeV}$ & $2056+i 37 \mathrm{MeV}$ \\
\hline$\rho N$ & $0.43-i 0.20$ & $0.16+i 0.15 \mathrm{MeV}$ & $0.27+i 0.25 \mathrm{MeV}$ \\
$K^{*} \Sigma$ & $1.70-i 0.53$ & $0.71-i 0.084 \mathrm{MeV}$ & $1.22-i 0.29 \mathrm{MeV}$ \\
\hline
\end{tabular}


Table 9. The properties of the dynamically generated resonance with isospin $I=3 / 2$ and spin $S=3 / 2$ and its possible PDG counterpart.

\begin{tabular}{lcccc}
\hline Pole positions & Name and $J^{P}$ & Status & Mass & Width \\
\hline $1603+i 72 \mathrm{MeV}$ & $\Delta(1700) 3 / 2^{-}$ & $* * * *$ & $1670-1750 \mathrm{MeV}$ & $200-400 \mathrm{MeV}$ \\
\hline
\end{tabular}

Table 10. Pole position and coupling constants to various channels of the resonance found in the $I=3 / 2$ and $S=3 / 2$ sector.

\begin{tabular}{cc}
\hline$g_{i}$ & $1603+i 72 \mathrm{MeV}$ \\
\hline$\rho N$ & $0.81-i 0.29$ \\
$K^{*} \Sigma$ & $0.30+i 0.11$ \\
\hline
\end{tabular}

\section{Summary}

We have studied the the vector meson- baryon octet interaction in a coupled channel unitary approach, and obtained the kernels between vector mesons and baryon octet from a summation of diagrams corresponding a vector meson exchange in the $t-$ channel, octet baryon exchange in $s-, u$ - channels, and a contact interaction. In the $S$-wave approximation, the scattering amplitudes are calculated by solving the relativistic Lippman-Schwinger equations of coupling channels.

Altogether 13 poles are observed in the complex plane of the total energy in the center of mass system in channels with different isospins and spins. Many of these poles are associated to known states in PDG, In the channel of isospin $I=1 / 2$ and spin $S=3 / 2$, the resonances $N(1875) 3 / 2^{-}$and $N(2120) 3 / 2^{-}$, which are split with the older data in the newest PDG review, ${ }^{1}$ are generated dynamically. Moreover, for some resonances in our prediction, there are not PDG counterparts for them, and this might give a stimulus to search experimentally for these resonance states.

\section{References}

1. J. Beringer et al. (Particle Data Group), Phys. Rev. D86, 010001 (2012).

2. M. Bando, T. Kugo, S. Uehara, K. Yamawaki and T. Yanagida, Phys. Rev. Lett. 54, 1215 (1985).

3. M. Bando, T. Kugo and K. Yamawaki, Phys. Rep. 164, 217 (1988).

4. M. Harada and K. Yamawaki, Phys. Rep. 381, 1 (2003).

5. U. G. Meissner, Phys. Rep. 161, 213 (1988).

6. H. Nagahiro, L. Roca, A. Hosaka and E. Oset, Phys. Rev. D 79, 014015 (2009).

7. R. Molina, D. Nicmorus and E. Oset, Phys. Rev. D 78, 114018 (2008).

8. L. Geng and E. Oset, Phys. Rev. D 79, 074009 (2009).

9. P. Gonzalez, E. Oset and J. Vijande, Phys. Rev. C 79, 025209 (2009).

10. S. Sarkar, B.-X. Sun, E. Oset, M. J. Vicente Vacas, Eur. Phys. J. A 44, 431-443 (2010).

11. E. Oset and A. Ramos, Eur. Phys. J. A 44, 445 (2010). 
12. K. P. Khemchandani, H. Kaneko, H. Nagahiro and A. Hosaka, Phys. Rev. D83, 114041 (2011).

13. L. J. Reinders, H. Rubinstein and S. Yazaki, Phys. Rept. 127, 1 (1985).

14. A. V. Anisovich, R. Beck, E. Klempt, V. A. Nikonov, A. V. Sarantsev and U. Thoma, Eur. Phys. J. A 48, 15 (2012).

15. M. Batinic, S. Ceci, A. Svarc and B. Zauner, Phys. Rev. C 82, 038203 (2010).

16. T. P. Vrana, S. A. Dytman and T. S. H. Lee, Phys. Rept. 328, 181 (2000). 